\title{
Physical Contagion Contact
}

National Cancer Institute

\section{Source}

National Cancer Institute. Physical Contagion Contact. NCI Thesaurus. Code C88118.

Exposure from touching an object, person, or body substance contaminated with a suspected or confirmed infectious agent. 